\title{
Corrigendum \\ The Neuropeptide Galanin and Variants in the GalR I Gene are Associated with Nicotine Dependence
}

\author{
Kia J Jackson, Xiangning Chen, Michael F Miles, JoLynne Harenza and M Imad Damaj
}

Neuropsychopharmacology (2012) 37, 2345; doi:10.1038/npp.2012.95

Correction to: Neuropsychopharmacology (2011) 36, 2339-2348; doi:10.1038/npp.2011.123; published online 27 July 2011

In this article, the database of Genotypes and Phenotypes $(\mathrm{dbGaP})$ data sets used in this study were improperly acknowledged and cited in the manuscript. Based on the terms of the dbGaP Data Use Certification agreement, the Prostate, Lung Colon, and Ovary Screening Trial data set should be cited as follows:

Landi MT, Chatterjee N, Yu K, Goldin LR, Goldstein AM, Rotunno $\mathrm{M}$ et al (2009). A genome-wide association study of lung cancer identifies a region of chromosome $5 \mathrm{p} 15$ associated with risk for adenocarcinoma. Am J Hum Genet (2009) 85:679-691.

In addition, the following data sets should be included in the Acknowledgements as follows:

Study of Addiction: Genetics and Environment (SAGE): Funding support for the SAGE was provided through the NIH Genes, Environment, and Health Initiative (GEI) (U01 HG004422). SAGE is one of the genome-wide association studies (GWASs) funded as part of the Gene Environment Association Studies (GENEVA) under GEI. Assistance with phenotype harmonization and genotype cleaning, as well as with general study coordination, was provided by the GENEVA Coordinating Center (U01 HG004446). Assistance with data cleaning was provided by the National Center for Biotechnology Information. Support for the collection of data sets and samples was provided by the Collaborative Study on the Genetics of Alcoholism (U10 AA008401), the Collaborative Genetic Study of Nicotine Dependence (P01 CA089392), and the Family Study of Cocaine Dependence (R01 DA013423). Funding support for genotyping, which was performed at the Johns Hopkins University Center for Inherited Disease Research, was provided by the NIH GEI (U01HG004438), the National Institute on Alcohol Abuse and Alcoholism, the National Institute on Drug Abuse, and the NIH contract 'High throughput genotyping for studying the genetic contributions to human disease' (HHSN268200782096C). The data sets used for the analyses described in this manuscript were obtained from http://www.ncbi.nlm.nih.gov/gap through dbGaP accession number phs000092.v1.p.

GWAS of Schizophrenia: Funding support for the GenomeWide Association of Schizophrenia Study was provided by the National Institute of Mental Health (R01 MH67257, R01 MH59588, R01 MH59571, R01 MH59565, R01 MH59587, R01 MH60870, R01 MH59566, R01 MH59586, R01 MH61675, R01 MH60879, R01 MH81800, U01 MH46276, U01 MH46289 U01 MH46318, U01 MH79469, and U01 MH79470) and the genotyping of samples was provided through the Genetic Association Information Network. The data sets used for the analyses described in this manuscript were obtained from the dbGaP found at http://www.ncbi.nlm.nih.gov/gap through dbGaP accession numbers phs00013 and phg00013. Samples and associated phenotype data for the GenomeWide Association of Schizophrenia Study were provided by the Molecular Genetics of Schizophrenia Collaboration (PI: Pablo V Gejman, Evanston Northwestern Healthcare and Northwestern University, Evanston, IL, USA).

Lung cancer data set: Funding support for the GWAS of Lung Cancer and Smoking was provided through the NIH GEI (Z01 CP 010200). The human subjects participating in the GWAS were derived from The Environment and Genetics in Lung Cancer Etiology case-control study and the Prostate, Lung Colon, and Ovary Screening Trial, and these studies are supported by intramural resources of the National Cancer Institute. Assistance with phenotype harmonization and genotype cleaning, as well as with general study coordination, was provided by the GENEVA Coordinating Center (U01 HG004446). Assistance with data cleaning was provided by the National Center for Biotechnology Information. Funding support for genotyping, which was performed at the Johns Hopkins University Center for Inherited Disease Research, was provided by the NIH GEI (U01HG004438). The data sets used for the analyses described in this manuscript were obtained from dbGaP at http://www.ncbi.nlm.nih.gov/gap through $\mathrm{dbGaP}$ accession number phs000093. 